\title{
Automated Medication System for Rural and War Affected Areas
}

\author{
${ }^{1}$ Asif Ali Laghari, ${ }^{2}$ Intesab Hussain Sadhayo and ${ }^{3}$ Muhammad Ibrahim Channa \\ ${ }^{1,3}$ Department of Information Technology, QUEST, Nawabshah, Pakistan; \\ ${ }^{2}$ Department of Computer System Engineering, QUEST, Nawabshah, Pakistan; \\ asifalilaghari@gmail.com; intesab@quest.edu.pk; ibrahim.channa@quest.edu.pk
}

\begin{abstract}
Robot is machine like human beings working in hazardous situations, replace domain experts and provide accurate results. We proposed automated medication system that work like human physician experts in remote locations, where flood and thunderstorm occurs. It measure patient's temperature and blood pressure using sensors and get input from user via keyboard and voice system. After finding diseases, it retrieves symptoms for diseases from stored databases. The proposed automated medication system provides facility to people which are unable to reach big cities where medical facilities are available.
\end{abstract}

Keywords: Automated medication system; robot doctor; flood; thunderstorm; war.

\section{Introduction}

Today robotic technology is useful for work in difficult and critical situations where risk of human life is high. Experts from various fields refuse to work in challenging situations due to possible accident such as human life. They prefer to live risk free normal in metropolitans. In modern times artificial intelligence has made machines useful enough to work almost like human beings. The facilities for humans are easily accessible in major cities where as in rural areas their access is problem because of the distance of rural areas from major cities. Due to insufficient medical treatment resources, health is other major problem in rural areas. In developed countries health services are cheap and easily available on doorsteps. In least developed or war affected countries, it is hard to find a medical specialist. Sometimes thunderstorm or flood situations in countries make life difficult and different diseases are taken places so there is need for doctor to treat patients. Rural areas are away from capital cities and take doctors long time to reach patients for treatment and they have no communication in rural areas. Mostly children, elderly people and pregnant women lose their life. The situation worsens during floods. All roads become disconnected to reach major cities and outbreak of diseases like malaria, diarrhea and fever is occurred which causes mortality in children and elderly people. Therefore it is very difficult for rescue team to reach every patient. According to our research, the solution of this problem is the development of automated medication system that can work on behalf of expert doctors in difficult situations where flood or heavy thunderstorm destroy the area or in case war is held. There is need is to develop an automated medication system that serves in challenging situations to provide facilities to human beings. 
Robot is a machine, which contains sensors, manipulator, control system, database and software that forces machine to take sense or observe the environment, and make decisions like experts to solve the problems. "The term robotics encompasses a variety of research subareas, systems, and applications that span navigation; manipulation; walking, running, and flying; legged, wheeled, and tracked locomotion; autonomous underwater, aerial, and space vehicles; and medical, service, and automation systems"[1]. One subarea of robotic termed as social robots that help for social problems like eye contact, body language and also help to speech and gestures. Now robots share part in human life the mobile robots are used for guide people in museums and robots are also use for driving vehicles few applications of robots use in Physics (nanotechnology) [2]. "NASA tests robots for exploration in areas called analogs. Analogs are places where the environment is similar to locations like Mars or the moon, where a robot may be used. One NASA analog is in the Arizona desert. NASA robotics experts conduct field tests in the desert to assess new ideas for rovers, spacewalks and ground support. Some of these tests are conducted by a team called Desert RATS, which stands for Desert Research and Technology Studies" [3]. The main reason of development of robots for reduce heavy work force from humans and shift that work on machines. Three are two common types of robots used in industries:

1. Fixed robots. 2. Mobile robots.

Fixed robots are usually fixed at one place in industries, where they work as pick and drop things from one assembly line to other assembly line. Robotic arms are used to hold materials at working hours. Mobile robots are more intelligent and have more functionality compared to fixed robots because mobile robots can move from one place to other place. Some examples of robots are, a dancing robot and News anchor robot both developed by Japan. These robot uses sensor, vision, motion and detecting function to work properly in environment. Main components used in robot for performing operations are robotic arm, controller, end effectors, drives, manipulators, and sensors.

Robotic Arm: Robotic arm is used in industrial robots for holding materials. Robotic arm moves in six axes to perform operations, some robotic have less depend on applications where robots are used. The robotic arm also has several other components including wiring, cables, and drives. Typically, the larger the robotic arm, the higher the maximum allowable payload [9].

Controller: it is the brain of robot. It controls the robotic arm and all other operation which robot performs. User can input data and control for task by using controller of robot [10].

Drives: Drives are the servo-motors of robot which play part to move robot from one place to other place and move the robotic arm required direction in industry [11].

End Effectors: End effectors are made on the end of robot arm used as gripper or holding the materials. End effectors have many shapes, sizes and unique according to their applications like cutting, painting and welding [12].

Sensors: Sensors are environment sensing devices and reacting on the observations from surroundings. Sensors are uses in robots for visioning, quality control temperature, pressure, fire smoke detection and detecting current position in working area $[13,14]$.

Expert systems are the part of artificial intelligence which contains knowledge of domain experts for assist juniors in work. Experts system didn't collects data of user by using sensors but they only respond user's queries. The inference engine contains mechanism of expert system which follows the rules and 
Asif Ali Laghari, Intesab Hussain Sadhayo and Muhammad Ibrahim Channa; Automated Medication System for Rural and War Affected Areas, Transactions on Machine Learning and Artificial Intelligence, Volume 3 No 1 Feb, (2015); pp: 8-15

procedure a logical reasoning for answer. Current robot doctors (Robot machines) assist the physician and medical staff by taking automatically temperature, blood pressure, heartbeat rate and pulse rate. Robot doctors currently used in hospitals didn't provide any symptoms solution to patient in normal or emergency situations. The development of good decision system of robots that make accurate decision, provide accurate result in practical environment and provide facilities to patients to communicate easily or share information about their diseases easily. In this paper we propose automated medication system which is combination of expert system and new robot doctors. Automated medication system take input from user and automatically measure temperature and blood pressure of patient and provide accurate symptoms for treatment.

This paper is divided in IV sections, II section contains background of previous development in robotic technology, the III sections illustrate proposed automated medication system and finally we conclude work in section IV.

\section{Literature Review}

Robotic technology is used in hospitals for patient care, surgeons use for accurate surgeries of patients. In USA hospitals the paralyzed patients from neck to down have been able to control a robotic arm using their thoughts. In figure patient drink first time in nearly 15 years [4].

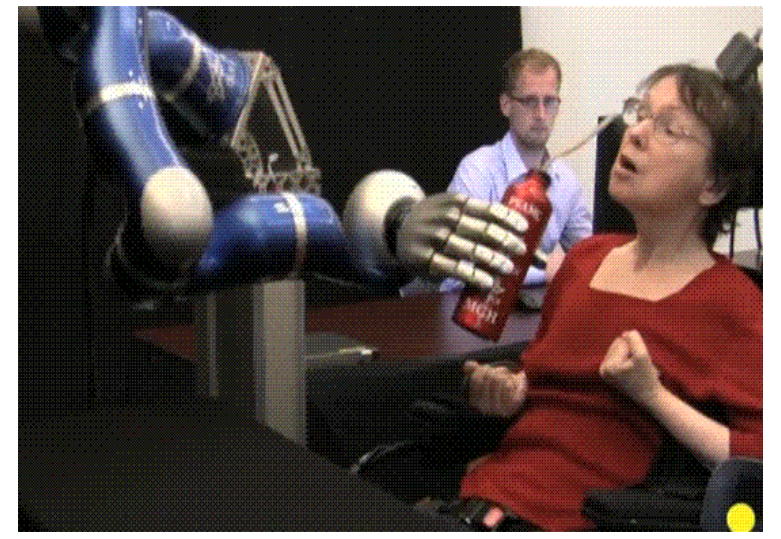

Figure 1: Paralyzed patient drinking using robotic arm

A researcher from USA designed wheelchair-mounted robotic arm (WMRA) for patients to meet the needs of mobility-impaired [5]. The development of this robotic wheelchair with robot arm provides help them to easily move things using robotic arm, they cannot move forward from wheel chair to open a door. Researcher also integrated brain computer interface $(\mathrm{BCl})$ this system measures the brain activities and transform them to control signals for the control of WMRA. In [6] Juan Fasola proposed spatial language communication model in robots for home usage of robots. Robots with natural language processing system work in home with non-expert users. This proposed model provides new research track that enables robots to communicate with non-expert people and have provide facility to them in home. Mirror Image Movement Enabler (MIME) robot developed by Peter for shoulder and elbow neuro-rehabilitation in sub-acute stroke patients [7]. Development of MIME robot provides good therapy results, it is cheap and no tiredness like therapist who continuously move the shoulder and elbow of patients. 
In Northern Ireland Telepresence, robot is used for patient health care. Telepresence robot will be used Daisy Hill hospital; it enables doctors to communicate with patients from remote locations using robot visual system. Robot can share huge data of patients with doctors in charts and graphs. Robot has functionality of stethoscope at the back of robot for listening the patient's lungs and heart [8]. In figure 2 design of robot is given.

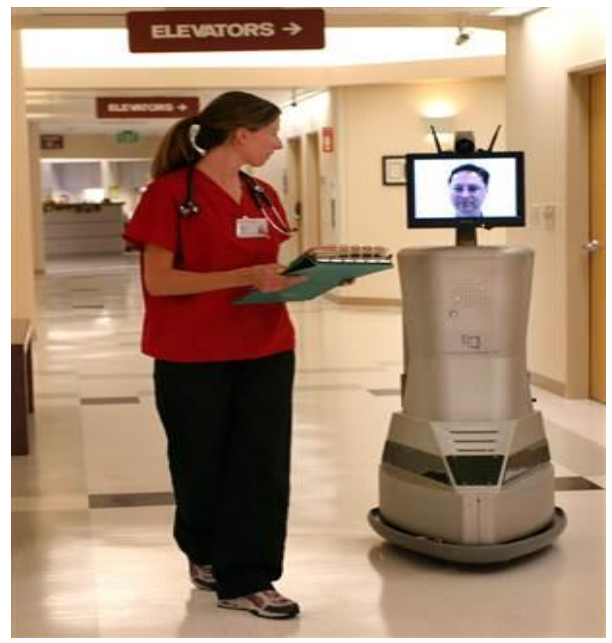

Figure 2: Telepresence Robot working in Daisy Hill hospital

Robots are used in so many other applications of health caring for provide facility to patients and make job easy for doctors. Robots are still controlled by operators for performing health care tasks but no standalone robot is developed yet which check patient and provide accurate medical symptom for cure his/her disease.

All previous automated medication system $s$ were made for different treatments and few of them only assist or collect data for doctors. The proposed architecture will be standalone robot system which works like expert physician doctor in rural areas or during floods and thunderstorm.

\section{Proposed Architecture of Automated Medication System}

In this paper, we proposed automated medication system for treatment of diseases which occurred in rural area when flood or thunderstorm occurred. The proposed automated medication system works like physician and assist patients for usage of symptoms for treatment cure from diseases. The proposed system depends on sensors, database, processing unit, storage, network connection and user interface (input \& output).

In development of automated medication system process we conduct interview and collect data from physician for diseases (fever, dehydration, Malaria, headache etc.) that occurred during the flood and thunderstorm. All information regards to diseases and treatments are store in database and MySQL database is used for automated medication system. Sensors are the main part of automated medication system which collects information in shape of body temperature of patient and blood pressure. Inference engine is responsible for process all operation from getting information from patient about their disease and find suitable treatment for patient. Automated medication system is connected via network connection to central hospital system for analysis of database and updating of database. User interface is used to help to patient communicate with robot system for input information in system. 
Asif Ali Laghari, Intesab Hussain Sadhayo and Muhammad Ibrahim Channa; Automated Medication System for Rural and War Affected Areas, Transactions on Machine Learning and Artificial Intelligence, Volume 3 No 1 Feb, (2015); pp: 8-15

Input and output system is an important part of automated medication system. Keyboard is used to input the data. All components of automated medication system are given in figure 3 .

User starts using automated medication system service to input of temperature and blood pressure. Sensors measure the body temperature and blood pressure of patient. Patient has option to use user interface to input disease or health problem that causes illness. System provides a unique ID for every patient and stores his all information in database for future usage. For example, patient enters data of diseases from keyboard then system find out keywords or fetches names of diseases from user's data. After finding the keyword of diseases, system software will find symptoms for every disease from database which depends on knowledge of ten (10) expert physicians. Final output of automated medication system will provide list symptoms for patient in printout on paper. All automated medication systems are connected to central system for sharing information of patient's facts and figures. After certain time period, all automated medication systems database will be updated with latest diseases and their treatment symptoms because every day new research in medical field find new symptoms for diseases which provides quickly relief to patient from illness.

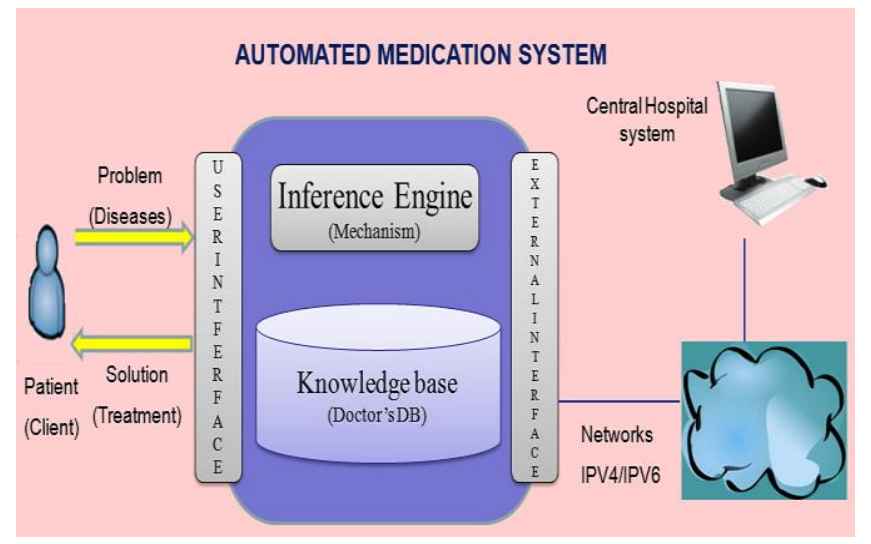

Figure 4: Proposed Automated Medication System architecture diagram

\section{Results and Discussion}

This section contains results of databases which include main database of automated medication system and Patient information database. The main database of automated medication system contains columns of disease, symptom and ratings. Disease columns stores information of all diseases and symptom columns stores data of symptoms and their types it's tablet or syrup. The rating shows number of experts prefer symptom out of 10 given in figure 4. The database tested with running different search queries like disease name, symptom name as well as ratings. Figure 5 is SQL query execution and figure 6 contain the result of SQL query.

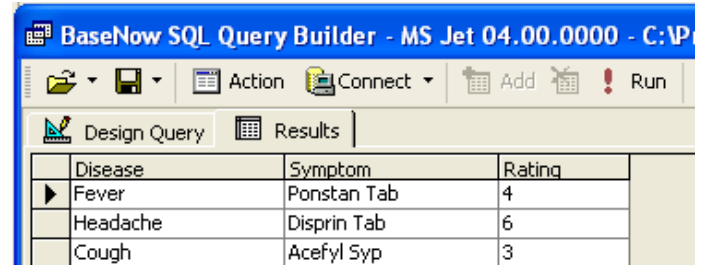

Figure 5: Snapshot of Automated Medication system DB 


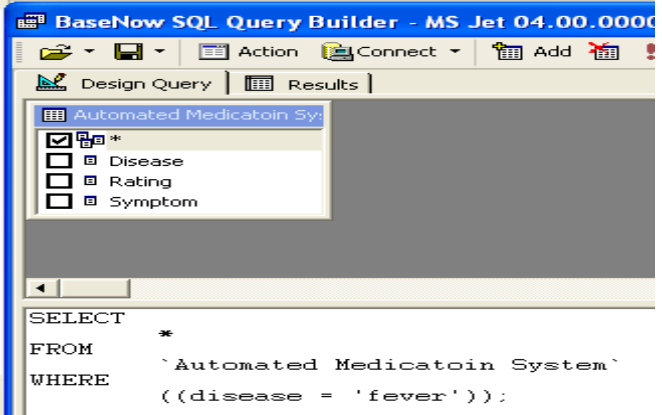

Figure 6: Snapshot of SQL Execution

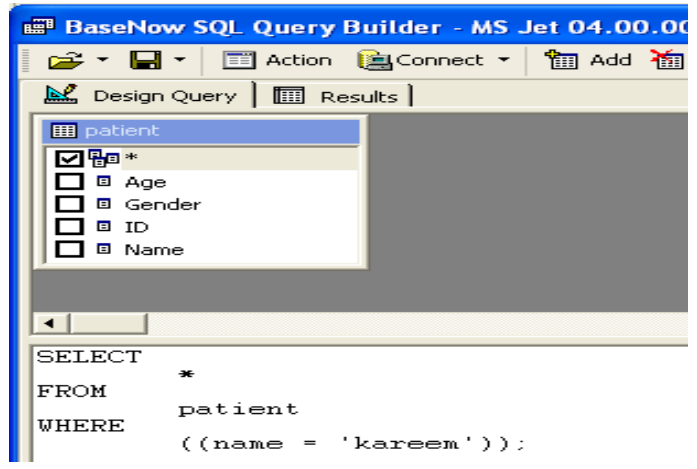

Figure 8: Snapshot of SQL query execution

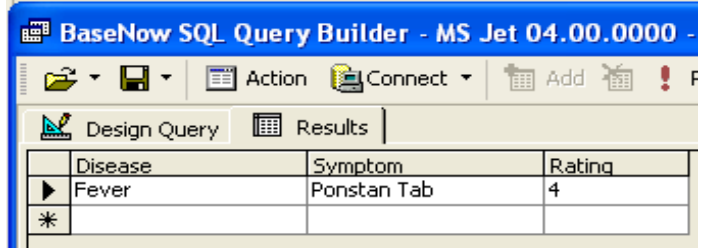

Figure 7: Snapshot of result

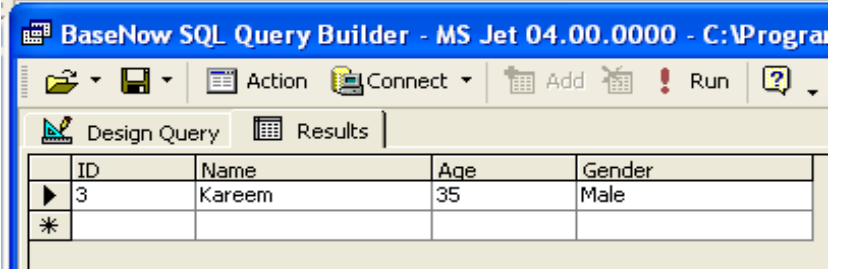

Figure 9: Snapshot of result of patient's table

The user interface is given for user input about diseases. The user interface is required three steps for symptoms required for treatment of the patients. In figure 10: user interface display automatically collected information of blood pressure and blood pressure of patient. The system automatically assigns ID to every new user for identification and future use for system. User will enter his details name, age, gender and diseases from the suffering and page also contain option for information submit or cancel and reset page.

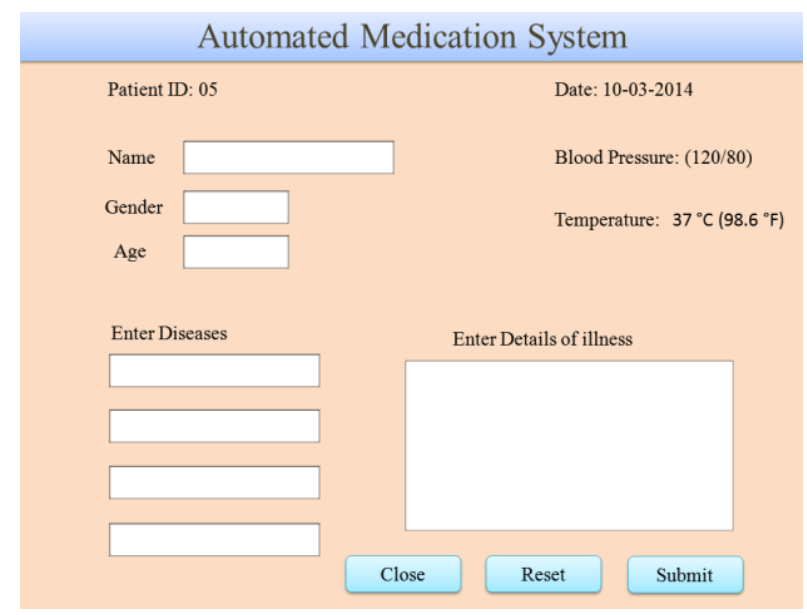

Figure 10: Snapshot of result of user interface

After the pressing the submit button in first user interface form given in figure $10 ., 2^{\text {nd }}$ user interface page will open. This page ask question from patient about the major diseases in figure 11. 


\section{Automated Medication System}

Do you have any major disease?

$\ulcorner$ Diabetes

$\ulcorner$ Blood Pressure

$\ulcorner$ Allergy (from any medicine )

$\ulcorner$ Any Disorder

$$
\text { Cancel }
$$

Continue

Figure 11: Snapshot of result of user interface

Finally system will display page contain symptoms of diseases which entered by patient and the time when symptoms can be taken.

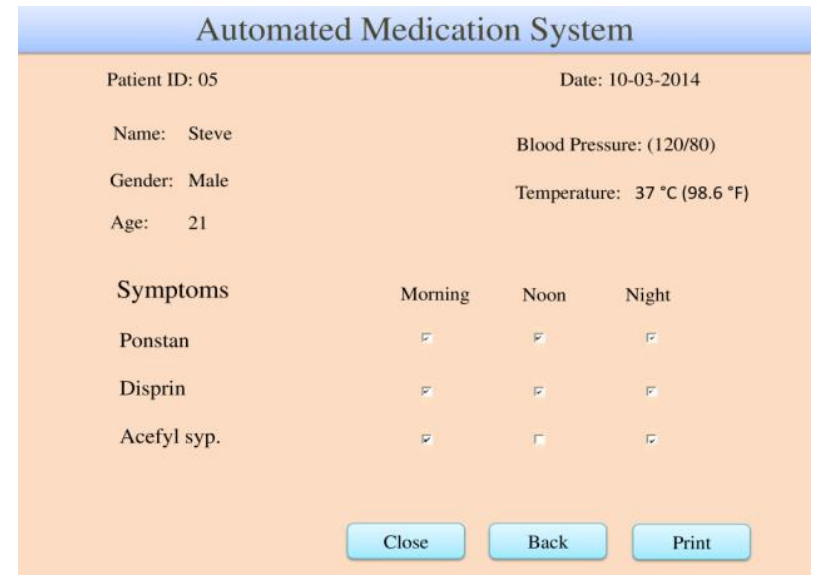

Figure 12: Snapshot of result of user interface

\section{Conclusion \& Future Work}

The proposed automated medication system is an integration of previous robot doctors, which assists the domain experts by taking temperature, blood pressure and hurt beat rate by adding features of expert systems. The proposed system is standalone system, which provides facility of first aid for the patients with less mobility in large hospitals during disasters, e.g., flood, thunderstorm and war situations.

\section{REFERENCES}

[1]. Scassellati, B., Admoni, H., \& Mataric, M. Robots for use in autism research. Annual Review of Biomedical Engineering, 14, 275-294. (2012)

[2]. Fraichard, T., \& Kuffner, J. J. Guaranteeing motion safety for robots.Autonomous Robots, 32(3), 173-175 (2012) 
[3]. http://www.nasa.gov/audience/foreducators/robotics/home/index.html last accessed [05/01/2013]

[4]. http://www.bbc.co.uk/news/health-18095179 last accessed [05/01/2013]

[5]. Palankar, M., De Laurentis, K. J., Alqasemi, R., Veras, E., Dubey, R., Arbel, Y., \& Donchin, E. Control of a 9-DoF wheelchair-mounted robotic arm system using a P300 brain computer interface: Initial experiments. In Robotics and Biomimetics, ROBIO 2008. IEEE International Conference on (pp. 348-353). IEEE. (2009)

[6]. Fasola, J., \& Matarić, M. J. Using Spatial Language to Guide and Instruct Robots in Household Environments. In 2012 AAAl Fall Symposium Series. (2012)

[7]. Lum, P. S., Burgar, C. G., Van der Loos, M., Shor, P. C., Majmundar, M., \& Yap, R. MIME robotic device for upper-limb neurorehabilitation in subacute stroke subjects: A follow-up study. Journal of rehabilitation research and development, 43(5), 631. (2006)

[8]. http://www.bbc.co.uk/news/uk-northern-ireland-19328560 last accessed [11/01/2013].

[9]. Li, T., Nakajima, K., Cianchetti, M., Laschi, C., \& Pfeifer, R. Behavior switching using reservoir computing for a soft robotic arm. In Robotics and Automation (ICRA), IEEE International Conference on (pp. 4918-4924). IEEE (2012)

[10]. Dalibard, S., Thalmann, D., \& Magnenat-Thalmann, N. Interactive design of expressive locomotion controllers for humanoid robots. InRO-MAN, 2012 IEEE (pp. 431-436). IEEE (2012)

[11]. Ahmed, F. I., Zaki, A. M., \& Ebrahim, E. A. Neuro-controller for high performance induction motor drives in robots. In Neural Networks, 2001. Proceedings. IJCNN'01. International Joint Conference on (Vol. 3, pp. 2082-2087). IEEE. (2001)

[12]. De Luca, A., Mattone, R., \& Oriolo, G. Dynamic mobility of redundant robots using end-effector commands. In Robotics and Automation, 1996. Proceedings. IEEE International Conference on (Vol. 2, pp. 1760-1767). IEEE (1996)

[13]. Murakami, K., Matsuo, K., Hasegawa, T., \& Kurazume, R. Position tracking and recognition of everyday objects by using sensors embedded in an environment and mounted on mobile robots. In Robotics and Automation (ICRA), 2012 IEEE International Conference on (pp. 22102216). IEEE (2012)

[14]. http://www.roboticsbible.com/uses-of-sensors-in-robotics.html 